\title{
Towards a slime mould-FPGA interface
}

\author{
Richard Mayne • Michail-Antisthenis \\ Tsompanas · Georgios Ch. Sirakoulis · \\ Andrew Adamatzky
}

Received: date / Accepted: date

\begin{abstract}
Purpose

The plasmodium of slime mould Physarum polycephalum is a multinucleate single celled organism which behaves as a living amorphous unconventional computing substrate. As an excitable, memristive cell that typically assumes a branching or stellate morphology, slime mould is a unique model organism that shares many key properties of mammalian neurons. There are numerous studies that reveal the computing abilities of the plasmodium realized by the formation of tubular networks connecting points of interest. Recent research demonstrating typical responses in electrical behaviour of the plasmodium to certain chemical and physical stimuli has generated interest in creating an interface between $P$. polycephalum and digital logic, with the aim to perform computational tasks with the resulting device.
\end{abstract}

\section{Methods}

Through a range of laboratory experiments, we measure plasmodial membrane potential via a non-invasive method and use this signal to interface the organism with a digital system.

\section{Results}

This digital system was demonstrated to perform predefined basic arithmetic operations and is implemented in a field-programmable gate array (FPGA). These basic arithmetic operations, i.e. counting, addition, multiplying, use data that were derived by digital recognition of membrane potential oscilla-

Richard Mayne* \& Andrew Adamatzky

Unconventional Computing Group, University of the West of England, Bristol, UK

*Corresponding author:

Tel.: +447979-522256

E-mail: richard.mayne@uwe.ac.uk

Michail-Antisthenis Tsompanas \& Georgios Ch. Sirakoulis

Laboratory of Electronics, Department of Electrical and Computer Engineering,

Democritus University of Thrace, Xanthi, GREECE 
tion and are used here to make basic hybrid biological-artificial sensing devices. Conclusions

We present here a low-cost, energy efficient and highly adaptable platform for developing next-generation machine-organism interfaces. These results are therefore applicable to a wide range of biological/medical and computing/ electronics fields.

Keywords Physarum polycephalum · Digital Electronics · Machine-organism Interface $\cdot$ Unconventional Computing

\section{Introduction}

The plasmodium of slime mould Physarum polycephalum (Fig. 1) (Phylum Mycetozoa, Class Myxogastria) exists as a macroscopic, multinucleate single celled protistic organism, when in its vegetative life cycle phase. Capable of a range of complex behaviour patterns which may be interpreted as computation - e.g. solving maze puzzles [1], dynamic optimization of its nutrient harvesting network $[2]-P$. polycephalum may be viewed as a living amorphous computing substrate and is used as a model organism for unconventional computing research.

A variety of slime mould electronic devices, such as:

- self-repairing wires with variable electrical properties [3,4],

- chemical sensors [5],

- optical colour sensors [6],

- tactile sensors [7],

- electrical oscillators [8],

- memristors (resistors with memory) [9],

- logical gates: micro-fluidic [10], electrical [11] and frequency based [12],

have been developed and unadulterated plasmodia may be adapted to perform a range of useful computation, e.g. planning travel networks $[13,14]$.

Certain features of slime mould physiology such as electrical excitability, memristivity and branching/stellate morphology are shared by mammalian neurons. Slime mould culture is, however, simple and low-cost when compared with cell culture methods and, furthermore, there are no ethical issues surrounding the experimental use of slime mould. It is, therefore, a valuable model organism for developing hybrid artificial-organic systems which may be eventually adapted into biomedical devices.

One of the major limitations of current slime mould computing devices is that their operation and outcome interpretation require a significant amount of human input. Most of them rely on implementing a system for stimulating the plasmodium (e.g. with light or chemoattractants) by hand before manually 
interpreting its consequent behaviour, such as migratory patterns, physiological state or bioelectrical phenomena. In this article, we demonstrate how the $P$. polycephalum plasmodium may be interfaced with conventional computing architectures to create hybrid devices which enable automated monitoring of plasmodial responses and provide a basis for developing computer-controlled plasmodial stimulation. We implement a range of basic arithmetic operations based on digital interpretation of plasmodial membrane potential and conclude by discussing the potential applications, limitations and open problems with this technology.

Bioelectrical potential was chosen as the slime mould 'output' to be received by the computer interface due to its ease of measurement and interpretation by electronic hardware. In its resting (unstimulated) state, plasmodial membrane potential oscillates rhythmically with a typical period of 1-2 minutes $[15,16]$ with an amplitude of $1-15 \mathrm{mV}$ when measured between anterior and posterior poles of the organism (Fig. 2) [4]. This oscillation in electrical potential approximately corresponds with the rhythmic physical oscillations of cytoplasm (shuttle streaming) which serve to distribute the cytoplasmic contents throughout the organism and generate motive force. This indicates that the electrical events in the plasmodium are a reflection of the physical processes which initiate shuttle streaming, e.g. $\mathrm{Ca}^{2+}$ influx/efflux to drive muscle protein contraction. Indeed, in Ref. [17], Adamatzky and Jones suggest that certain patterns in plasmodial activity may serve to communicate instructions between different parts of the organism. For a more comprehensive review of the electrical properties of the $P$. polycephalum plasmodium, please see Ref. [4].

Critically, membrane potential dynamics reflect the physiological state of the organism, and hence provide a basis for interpreting slime mould behaviour patterns. The $P$. polycephalum plasmodium has been demonstrated to respond to stimuli such as application of physical pressure [7], chemical gradients [5] and alterations in environmental temperature [18] in a characteristic fashion. Furthermore, morphological transformations into different life cycle forms also tend to be accompanied by an archetypal pattern of electrical activity [17]. The mechanisms underlying these responses are complex, but are essentially reflections of how the plasmodium responds to the stimulus. For example, attractants (such as moderate heat and nutrient sources $[19,18]$ ) and repellents (light, certain chemicals, substrate desiccation [20,21] etc.) will both tend to increase the frequency and amplitude of oscillation proportionally to the degree of attraction/repulsion, as these electrical phenomena are directly related to the mechanisms which cause taxis towards/away from the stimulus. A slime mould-computer interface is therefore a hypothetical possibility if the computer could be programmed to recognise the typical electrical responses of a plasmodium to a given stimulus and respond by initiating/halting sources of stimulation in the slime mould's environment.

An FPGA-based interface was chosen for this study, as FPGAs are recon- 


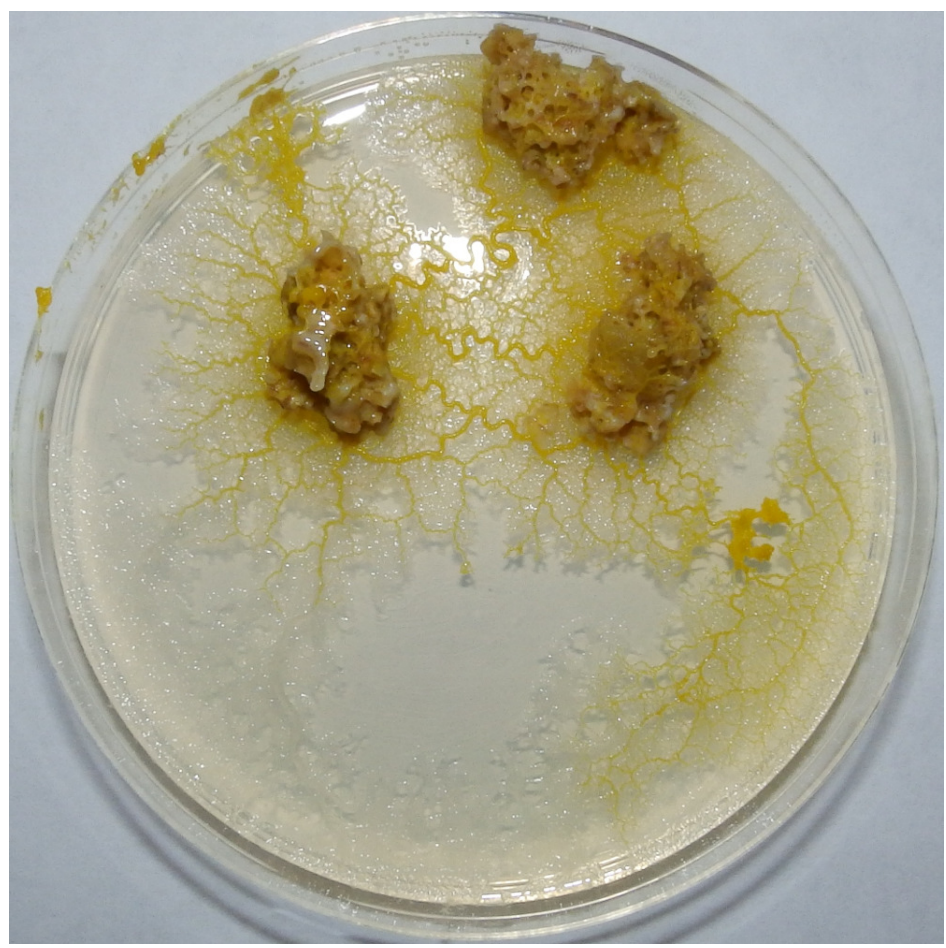

Fig. 1 Photograph of the $P$. polycephalum plasmodium propagating on a $2 \%$ non-nutrient agar plate, feeding on oats.

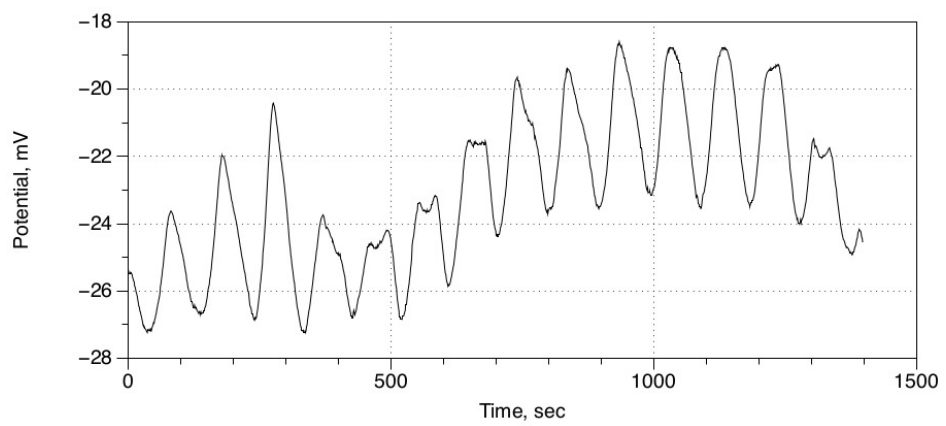

Fig. 2 Graph to show oscillations in plasmodial membrane potential when measured between the anterior and posterior poles of a $10 \mathrm{~mm}$ plasmodial tube, whilst in a vegetative (resting) state.

figurable devices capable of hosting complex and dynamically changing digital logic. FPGAs can be programmed to implement digital logic described by the user, thus, they are commonly used by the research community. Moreover, they are less expensive compared to application-specific integrated circuits (ASICs). Nonetheless, FPGA-based boards are easy to access and can implement a vast 
variety of digital applications. A simple system is designed here, in order to demonstrate the possible connection between the plasmodium and a digital computing system, which is based on an Altera's "Cyclone II FPGA Starter Board" [22].

Membrane potential was considered to be the most appropriate signal type to monitor the behaviour of the plasmodium, based on the results of a previous work [8]. An interface between the plasmodium and a digital system has been used in robot rooting applications [23]. However, the first generation of this interface, which is bidirectional, uses optical coupling of the plasmodium with the digital system. Taking into consideration the equipment and the algorithms needed for this interface, it can be characterized as an expensive and time consuming method. On the other hand, the second generation of this interface uses electrical characteristics of the plasmodium to extract information. More specifically, its impedance is measured by the application of an AC voltage to two segments of the plasmodium and comparing the difference of their amplitude and angle [24]. Nevertheless, the application of even a slight amplitude of voltage can alter the behavior of the plasmodium as realized in [8]. Consequently, measuring the membrane potential of the plasmodium in an unstimulated state is expected to be the best solution to map and analyse the computing behaviour concealed in the electrical signals transmitted by the plasmodium. This highlights the necessity to measure plasmodial bioelectrical properties in an entirely non-invasive way. Indeed, the results of Whiting et al. $[12,5]$ strongly support a non-invasive methodology for using slime mould as a chemical sensor.

\section{Methods}

P. polycephalum plasmodia were cultured on $2 \%$ non-nutrient agar (NNA) in $9 \mathrm{~cm}$ plastic Petri dishes in the absence of light at $22 \pm 2^{\circ} \mathrm{C}$. They were supplied with porridge oats as a nutrient substrate and were subcultured routinely every 2-4 days, as required. $10 \mathrm{~mm}^{2}$ samples of plasmodium were removed from their Petri dishes and inoculated onto a $1 \mathrm{ml}$ blob of NNA overlying a $90 \times 8 \mathrm{~mm}$ aluminium tape electrode (RS Components, UK) stuck to the base of an empty Petri dish. Another blob/electrode arrangement was present $10 \mathrm{~mm}$ away in the same dish and the plasmodium was left for $c .48$ hours to propagate across the gap, forming a $10 \mathrm{~mm}$ plasmodial tube linking the two electrodes (Fig. 3). Measurement of a single tube, as opposed to a tube network, was chosen to minimise the possibility of multiple discrete oscillatory patterns coexisting: it has been found in previous works $[3,4,12]$ that patterns of electrical activity are adequately repeatable despite morphological re-arrangement of the anterior and posterior poles of the organism, as long as a single tube of constant length links the two together. 


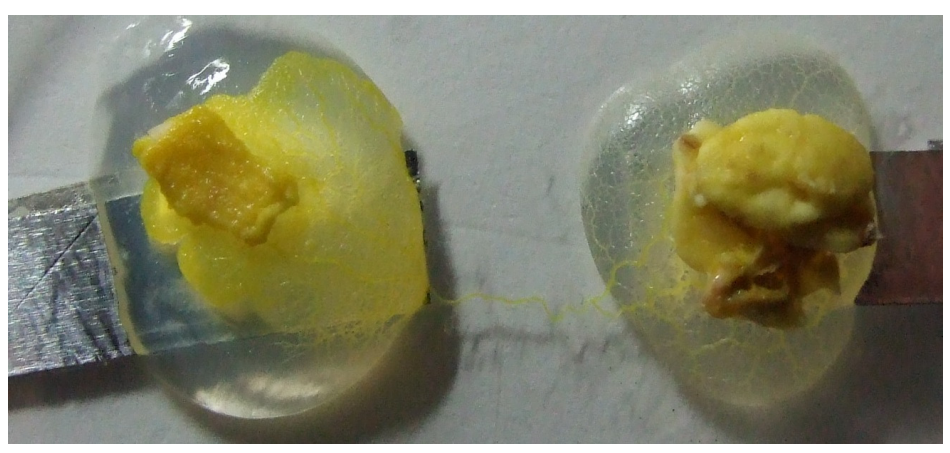

Fig. 3 Photograph of the experimental environment for growing and measuring the electrical properties of $10 \mathrm{~mm}$ plasmodial tubes.

The aluminium tape electrodes were connected to an Altera Cyclone II FPGA [22] via a custom-made external digitalization circuit (Fig. 4). The digitalization circuit is used in order to translate the plasmodial membrane potential, namely an analog signal, into a digital signal of one bit. More specifically, if the input potential is higher than a reference voltage the output of the circuit is high (or logic ' 1 '), while if the input is lower than the reference voltage the output is low (or logic ' 0 '). This can be realized by the schematic diagram of the digitalization circuit illustrated in Fig. 5. The reference voltage is set manually with a sensitive 25 -turn potentiometer (RS Components, UK) to alter the way in which the signal may be digitalized.

The digitalization circuit consists of two operational amplifiers: the first is used as a buffer and the second is used as a voltage comparator. The buffer is a voltage follower, granting isolation between the aluminium tape electrodes in contact with the plasmodium and the rest of the digital circuitry (FPGA). As the plasmodium is assumed to be in an unstimulated state, any fluctuation of conditions in its environment can act as input data that will alter its behaviour. Thus, measures were taken that the digital system will have the smallest possible interference to the plasmodium. This was achieved by utilising the aforementioned non-invasive methodology for measuring plasmodial electrical signals and by including the voltage follower in the digitalization circuit, which has a very high (near infinite) input impedance. Moreover, the comparator is used to detect if the input voltage is above or below a given threshold (the reference voltage), and therefore it performs in essence a 1-bit quantization. As there is no feedback to the inputs of the operational amplifier, its output will be nearly equal to the the positive power supply voltage when the input potential is higher than the reference voltage. On the other hand, when the input voltage is lower than the reference voltage, the output of the circuit should be the negative power supply voltage; however it will be equal to zero $(0 \mathrm{~V})$, due to the diode connected to the output pin of the circuit. 


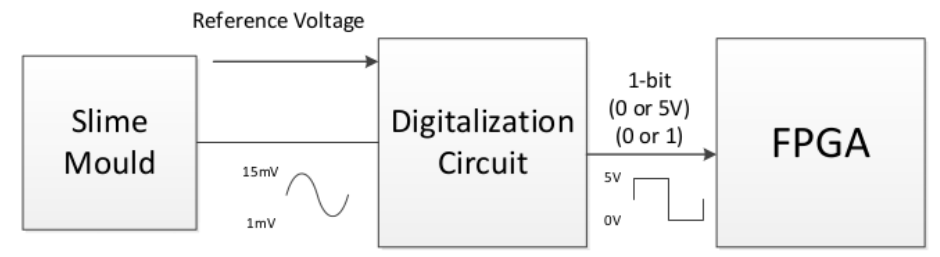

Fig. 4 Block diagram of the system.

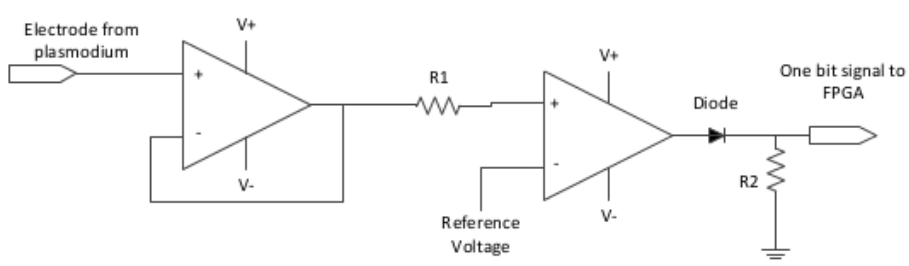

Fig. 5 External digitalization circuit with reference potential set to $0 \mathrm{~V}$.

The output of the digitalization circuit is connected to one pin of the input/output headers of the FPGA-based board. As a result the signal is rooted on the board and driven to a pin of the FPGA. The FPGA is programmed for the purpose of this study - to perform major basic arithmetic operations: counting, adding and multiplying. These functions are implemented into the FPGA and are initiated manually, by altering switches on the board. For the counting function, the input signal from the digitalization circuit, and hence from the plasmodium, is used as an enable signal. The counter, which increases the count by 1 for every millisecond that a low (0V) signal is applied to the FPGA pin, stops counting whenever an enable signal is present.

For the adding and the multiplying functions one operand is provided manually (using the board's switches) while the second operand is given by the plasmodium. The plasmodium is sampled three times, once every 50 seconds (an arbitrary amount of time that was selected based on previous laboratory observations on the oscillating behavior of the plasmodium), in order to provide the second operand in binary form starting with the least significant bit. The number provided by the plasmodium is of range 0 to 7 , because of the three time intervals that correspond to three bits representing a number. Conversely to the counter, the adder and multiplier functions will only acquire a value from the slime mould when an enable signal is present during the sampling interval. Let $a, b$ and $c$ be the three samples from the plasmodium in times $t=0 \mathrm{~s}, 50 \mathrm{~s}$ and $100 \mathrm{~s}$, respectively, from the initiation of the function, i.e. $a=1$ if input signal for $t=0 s$ is high and $a=0$ if input signal is low. The maximum and minimum values are acquired by Eq. 1 . 


$$
\begin{aligned}
& \min \left[\left(a * 2^{0}\right)+\left(b * 2^{1}\right)+\left(c * 2^{2}\right)\right]=0 \\
& \max \left[\left(a * 2^{0}\right)+\left(b * 2^{1}\right)+\left(c * 2^{2}\right)\right]=7
\end{aligned}
$$

The implementation of the logic on the FPGA can be designed as a digital circuit with specific inputs and outputs. However, here the Altera's Nios II processor is used, which is a soft processor that can be instantiated on an Altera FPGA device and programmed to perform a user-defined algorithm. The algorithm that is executed by the processor reads the state of the switches on the FPGA board and, depending on their state, executes one of the procedures (counting, adding or multiplying). Although the solution of a soft processor is not as fast as a fully custom digital circuit representation, the execution time for this specific application is not critical. Nonetheless, one of the advantages of a soft processor is that the synthesis of the system is more straightforward and the algorithms executed are written in high level programming languages. These characteristics allow the system to be produced in a shorter time period. Moreover, the inputs to the system (outputs of the plasmodium) can be saved or even sent to a conventional personal computer for further data processing.

Initial experimentation consisted of testing the efficacy of the interface by using the counter function to approximate the period of membrane potential oscillation. Initially, this involved connecting a plasmodial tube to the digitalization circuit and measuring membrane potential from the output of the buffer with a Picotech ADC-24 data logger (Pico UK) to ascertain whether or not the plasmodium was suitable for testing. Plasmodia that were not oscillating in a stable, rhythmic manner were excluded on the basis that they were not 'at rest' (i.e. stimulated). After approximately 10 minutes of measurement, the average membrane potential and mean oscillatory period were recorded. The digitalization circuit's reference voltage was then adjusted to the mean membrane potential and the FPGA was initialized with the counter setting. After waiting for the counter to stop and re-start, the time the counter was "active" (i.e. counting) was logged. This value was then doubled and compared to the previously measured value for oscillatory period, the rationale being that the counter would only function for the half-wave during which the membrane potential was lower than the reference voltage.

The adder and multiplier functions were used to implement a basic slime mould-based tactile sensor. In Ref. [7], Adamatzky found that applying a small amount of pressure from a light-weight mass such as a glass capillary tube to a $10 \mathrm{~mm}$ plasmodial tube connected to a data logger caused characteristic 'spikes' in membrane potential similar in appearance to action potentials in mammalian neurons; it was upon this principle that the adder and multiplier functions were evaluated in this study. Experiments began by measuring the membrane potential for $c$. 10 minutes with a data logger as in previous experiments, but the reference voltage was subsequently adjusted to a value approximately $5 \mathrm{mV}$ higher than the peak oscillatory voltage. The value 


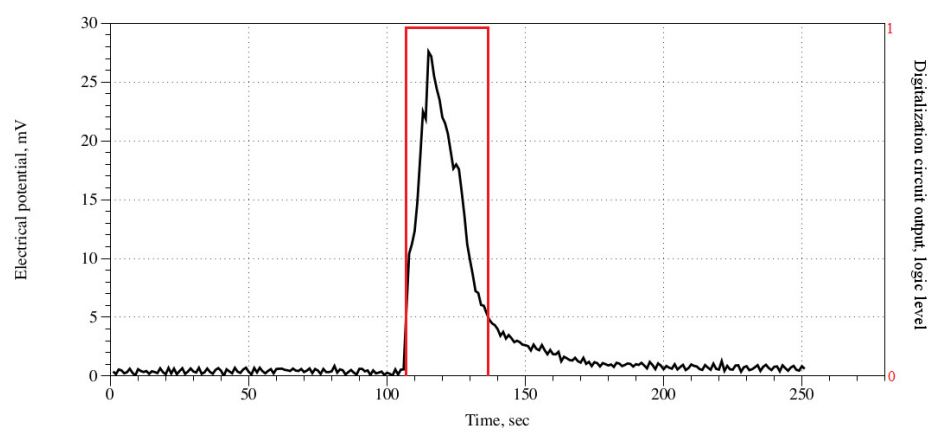

Fig. 6 Graph to show typical electrical response of plasmodial membrane potential to tactile stimulation with a $0.1 \mathrm{~g}$ glass capillary rod. The plasmodium was stimulated at 105 seconds. Note the characteristic 'spike' in response to stimulation, which drops to below the threshold value in under 50 seconds - i.e. the sampling interval. The corresponding output of the digitalization circuit is overlaid in red.

PeakVoltage $+5 \mathrm{mV}$ was chosen based on scoping experiments in which a plasmodial tube connected to a data logger had a short glass capillary tube weighing approximately $0.1 \mathrm{~g}$ gently laid across the center of the tube in a transverse orientation, which was then left in situ for the duration of the experiment. Our results were found to be consistent with those in Ref. [7] in that tactile stimulation caused spikes in membrane potential, usually far exceeding $10 \mathrm{mV}$ (see Fig. 6 for an exemplar plot). The spiking phase was found to occasionally lead into a slightly-hyperpolsarised refractory state which, crucially, returned to sub- $5 \mathrm{mV}$ values within about 40 seconds (mean $37 \mathrm{~s}, \mathrm{n}=10$ ) before receding into standard oscillation. The tactile sensor presented here therefore works by the principle that when the spike in membrane potential exceeds the reference voltage, an enable signal is generated, which is perceived by the FPGA if the sample window occurs during this stimulated phase. When the experiment is complete, if the number displayed by the FPGA is higher than the user-set value, this implies that tactile stimulation of the plasmodium has been detected by the FPGA. Experimentally, the user-defined value was arbitrarily selected as ' 2 ' and the plasmodium was left unstimulated for the first sampling window. The plasmodium was then stimulated $c$. 10 s before the second sampling window. No further intervention was performed for the rest of the experiment. The expected numerical outcome of these experiments were therefore $4(2+2$ and $2 \times 2)$, to correspond with the generation of an enable signal during the second sampling window.

\section{Results}

The counter function was experimentally found to function as intended in that the counter was observed to start and stop rhythmically in time periods approximating the pre-measured membrane potential oscillation period. The experimental setup is shown in Fig. 7. The approximation of the period was 


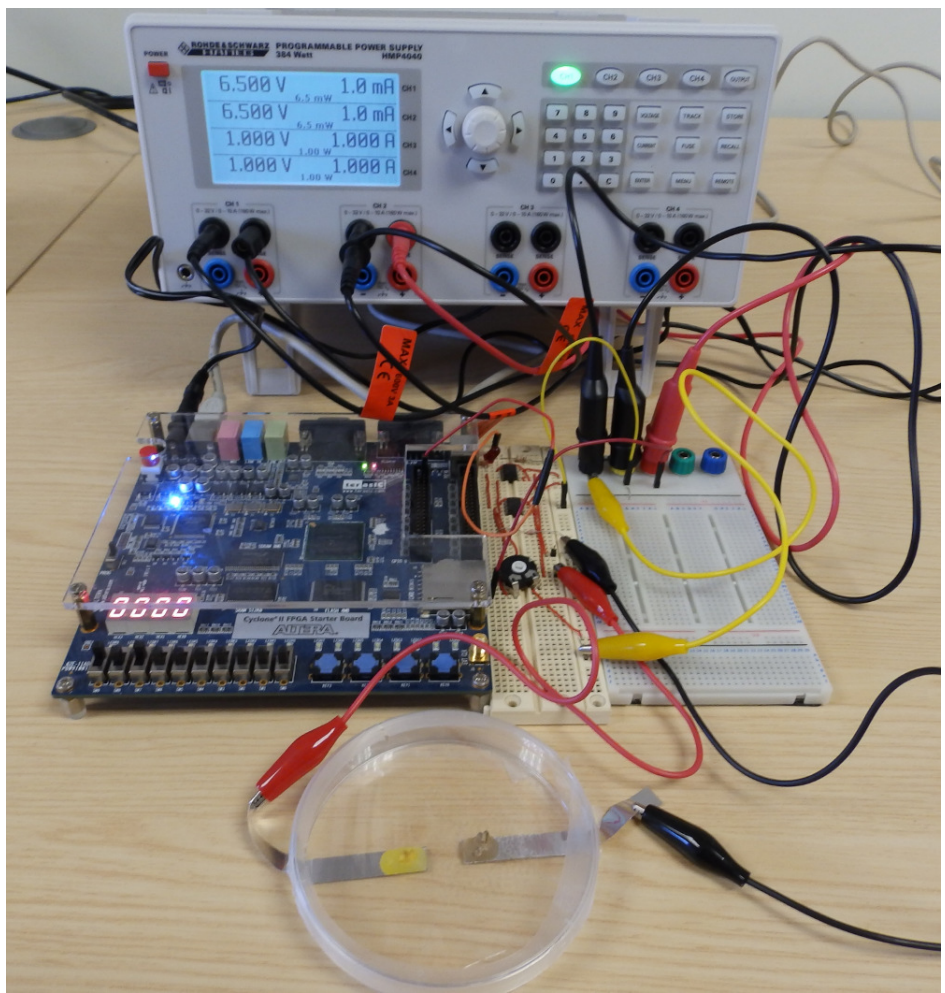

Fig. 7 Photograph of the experimental setup used. Results of the experiments may be interpreted via the FPGA's (left board) 4-figure numerical display (currently reading '0000'). The experimental plasmodium (lower) is linked to the FPGA by the digitalization circuit (centre board).

not statistically reliable, although results were typically within a \pm 10 second range of the measured value $(n=10)$. Furthermore, a boundary phase in which the transition between the counter turning on and off was observed in most samples during which the counter would intermittently stop and re-start. This period typically lasted for several seconds.

Both adder and multiplier functions were also found to function as intended. The outcomes were $90 \%$ and $80 \%$ success rate for the adder and multiplier, respectively $(\mathrm{n}=10$ each).

\section{Discussion}

We have here demonstrated a basis by which FPGA-living organism interaction may be implemented. Whilst this system currently has a limited range of practical applications - indeed, the biological component performed no 'computation' and communication between machine and organism was unidi- 
rectional - it provides a basic but highly adaptable system for developing more complex devices and represents a key milestone in the design of biological computing devices.

The inaccuracies in the counting function were likely a reflection of the limitations of the given setup, including response times of the hardware, sensitivity of the reference voltage potentiometer and the variability of the biological component: this highlights the inherent variability in living systems and hence the need for stringent optimisation and extensive repetition of experiments when fabricating hybrid living-artificial electronic systems.

With specific regards to slime mould computing, we have demonstrated the efficacy of using $P$. polycephalum as a dynamic sensing element. Further exploration of this technology could result in the development of devices which enable bidirectional communication between machine and organism via an integrated system for stimulating the plasmodium, e.g. light sources, heating/cooling elements. Such a system could be used for the implementation of learning or classification algorithms. This could, in turn, be used to model learning and problem solving in other cell types, e.g. neurons.

The fact that slime mould may be easily adapted into such a system would imply that other types of cell could also be used in a similar investigation due to the similarities between slime mould and mammalian cells (as mentioned in the introduction). Allowing for adaptation of the experimental environment e.g. sample environment, microelectrode design and filtering of the measured electrical signals — we have presented here a digital electronics interfacing platform which may be applied to tissue slices or even cultured cells. The possibilities of such a cost-effective, adaptable platform that readily facilitates cross-disciplinary work between biomedical and electrical/computing fields are enthusing when considering the potential outcome of such investigations. More specifically, we suggest that such a system would be a viable development platform for integrated biomedical systems, e.g. neurally-integrated prosthetics, implanted blood chemistry sensors.

To pre-empt criticisms of the results presented here and highlight areas for further study, it is pertinent to address some of the most obvious limitations the prototype system employed in this investigation by way of a conclusion. Only one plasmodial tube was employed, meaning that the system has only a one bit capability. This is a major confining factor because one bit can only be used in a limited number of operations. An aspect of future work could be the multiple bit quantization of the input signal in order to obtain more precise values of the membrane potential oscillations. Moreover, through the use of multiple electrodes connected by a complex multi-tube plasmodial network, a system of several bits could be implemented. Such a signal can be analysed in order to map the behaviour of the network formed. These alterations would require a slightly different digitalization circuit (using several voltage com- 
parators) and some changes in the programming configuration of the FPGA. These changes could also be implemented for different cell types to the same effect.

For such enhancements to be implemented, the reliability of the devices - i.e. their ability to continue functioning after repeated stimulation - must necessarily be enhanced. Morphological adaptation of the plasmodium is one of the major factors in reducing device reliability as rearrangements of the plasmodial tube network inevitably occur as a result of repeated stimulation. This tends to cause either breakage of the tube or the formation of a new morphology whose electrical characteristics are not consistent with those of the original tube. Future work should therefore focus upon using less insulting forms of plasmodial stimulation and employing techniques to reduce the speed/frequency of morphological adaptation. For example, the authors have observed that the rate of plasmodial migration is heavily dependent on temperature: indeed, it is a common practice amongst slime mould researchers to refrigerate plasmodia that have grown into desirable patterns but will not be immediately used, as this tends to prevent network rearrangement without causing noticeable deleterious effects to the health of the plasmodium. It may therefore be profitable to explore cooling the experimental environment to a constant temperature that preserves plasmodial life-processes but slows movement. It should be noted, however, that patterns of plasmodial electrical activity will almost certainly be 'non-normal' at sub-normal temperatures.

\section{Appendices}

\subsection{Declaration of Interest}

The authors declare no conflict of interest.

Acknowledgements The authors gratefully acknowledge funding from the European Union Comission's Seventh Framework Programme (grant agreement number 316366, "Physarum Chip").

\section{References}

1. T. Nakagaki, H. Yamada, A. Tóth, Nature 407(6803), 470 (2000)

2. A. Adamatzky, Bioevaluation of world tranport networks (World Scientific, 2012)

3. A. Adamatzky, Biomed Eng Lett 3, 232 (2013)

4. R. Mayne, A. Adamatzky, Nano LIFE 4(1450007) (2014). DOI 10.1142/S179398441450007X

5. J. Whiting, B. Costello, A. Adamatzky, Sens Actuators B: Chem 191, 844 (2014)

6. A. Adamatzky, Org Electron 14(12), 3355 (2013)

7. A. Adamatzky, Sens Actuators B: Chem 188, 38 (2013)

8. A. Adamatzky, Microelectron Eng 124, 58 (2014)

9. E. Gale, A. Adamatzky, B. Costello. Are slime mould living memristors? (2014). ArXiv: 1306.3414

10. A. Adamatzky, T. Schubert, Mater Today 17, 86 (2014) 
11. R. Mayne, A. Adamatzky, Int J Gen Sys pp. 37-41 (2015). DOI 10.1080/03081079.2014.997528

12. J. Whiting, B. Costello, A. Adamatzky, Biosyst 124, 21 (2014)

13. A. Adamatzky, IEEE Trans Cybern 44, 126 (2014)

14. A. Tero, S. Takagi, T. Saigusa, K. Ito, D. Bebber, M. Fricker, K. Yumiki, R. Kobayashi, T. Nakagaki, Science 327(5964), 439 (2010)

15. U. Kishimoto, J Gen Physiol 41(6), 1205 (1958)

16. K. Wohlfarth-Bottermann, J Exp Biol 67, 49 (1977)

17. A. Adamatzky, J. Jones, Biophys Rev Lett 06, 29 (2011)

18. D. Knowles, M. Carlilie, J Gen Microbiol 108(1), 9 (1978)

19. A. Durham, E. Ridgway, J Cell Biol 69, 218 (1976)

20. Y. Kakiuchi, T. Takahashi, A. Murakami, T. Ueda, Photochem Photobiol 73(3), 324 (2001)

21. Y. Nakagaki, H. Yamada, T. Ueda, Biophysical Chemistry 82(1), 23 (1999)

22. Altera Corporation. Cyclone ii fpga starter development board reference manual (2006)

23. S. Tsuda, S. Artmann, K.P. Zauner, in Artificial Life Models in Hardware, ed. by A. Adamatzky, M. Komosinski (Springer London, 2009), pp. 213-232

24. H.G. Coster, T.C. Chilcott, A.C. Coster, Bioelectrochem Bioenerg 40(2), 79 (1996) 